\title{
On strongly $\alpha-I$-Open sets and a new mapping
}

\author{
R.Devi, A.Selvakumar, M.Parimala \\ Department of Mathematics, \\ Kongunadu Arts and Science College, \\ Coimbatore - 641 029, Tamilnadu, India. \\ email: rdevicbe@yahoo.com \\ and \\ S.JAFARI \\ College of Vestsjaelland Syd, \\ Herrestraede 11,4200 Slagelse, Denmark.
}

\begin{abstract}
In this paper, we introduce the notion of strongly $\alpha$-I-open sets in ideal topological spaces and investigate some of their properties. Further we study the continuous functions for the above set and derive the some of their properties.
\end{abstract}

\section{RESUMEN}

En este trabajo, se introduce la noción del gran conjunto $\alpha$-I-abierto ideal en espacios topológicos y se investigan algunas de sus propiedades. Además se estudian las funciones continuas para el conjunto y parte de sus propiedades.

Keywords: $\alpha$ - $I$-open set, Strongly $\alpha-I$-open set and $B_{I}$ set.

Mathematics Subject Classification: 54A05,54D10,54F65,54G05. 


\section{Introduction}

The notion of $\alpha$-open sets was introduced and investigated by Njastad [16]. By using $\alpha$-open sets. Mashhour et al. [14] defined and studied $\alpha$-continuity and $\alpha$-openness in topological spaces. Ideals in topological spaces have been considered since 1930. This topic has won its importance by the paper of Vaidyanathaswamy [19]. In 2002, Hatir and Noiri [6] have introduced the notion of $\alpha$-I-continuous functions and used it to obtain a decomposition of continuity. The notion of $B_{I}$ sets introduced by Hatir and Noiri [6] and provided a decomposition of continuity. In this paper, we introduce strongly $\alpha$ - $I$-open sets and establish a decomposition of continuity.

In 1990, Jankovic and Hamlett [9] introduced the notion of $I$-open sets in ideal topological spaces. An ideal is defined as a non-empty collection $I$ of subsets of $X$ satisfying the following two conditions. (1) If $A \in I$ and $B \subset A$, then $B \in I$. (2) If $A \in I$ and $B \in I$, then $A \cup B \in I$. An ideal topological space is a topological space $(X, \tau)$ with an ideal $I$ on $X$ and it is denoted by $(X, \tau, I)$. For a subset $A \subset X, A^{*}(I)=\{x \in X: U \cap A \notin I$ for each neighbourhood $U$ of $x\}$ is called the local function of $A$ with respect to $I$ and $\tau$ [9]. We simply write $A^{*}$ instead of $A^{*}(I)$ to be brief. For every ideal topological space $(X, \tau, I)$, there exists a topology $\tau^{*}(I)$, finer than $\tau$, generated by $\beta(I, \tau)=\{U-i: U \in \tau$ and $i \in I\}$, but in general $\beta(I, \tau)$ is not always a topology [9]. Additionally, $c l^{*}(A)=A \cup A^{*}$ defines a kuratowski closure operator for $\tau^{*}(I)$. Given a space $(X, \tau, I)$ and $A \subset X, A$ is called $I$-open if $A \subset \operatorname{int}\left(A^{*}\right)$ and a subset $K$ is called $I$-closed if its complement is $I$-open $[8,9]$.

\section{Preliminaries}

First we will recall some definitions used in sequel.

Definition 2.1. A subset $A$ of an ideal topological space $(X, \tau, I)$ is said to be

1. $\alpha$-I-open [6] (resp. $\alpha$-open [16]) if $A \subset \operatorname{int}\left(c^{*}(\operatorname{int}(A))\right)(\operatorname{resp.} A \subset \operatorname{int}(\operatorname{cl}(\operatorname{int}(A))))$,

2. semi-I-open [6] (resp. semi-open [12]) if $A \subset \operatorname{cl}^{*}(\operatorname{int}(A))(\operatorname{resp} . A \subset \operatorname{cl}(\operatorname{int}(A)))$,

3. pre-I-open [1] (resp. pre-open [13]) if $A \subset \operatorname{int}\left(c l^{*}(A)\right)(\operatorname{resp} . A \subset \operatorname{int}(\operatorname{cl}(A)))$,

4. b-I-open [3] (resp. b-open [2]) if $A \subset \operatorname{int}\left(c l^{*}(A)\right) \cup c l^{*}(\operatorname{int}(A))(\operatorname{resp.} A \subset \operatorname{int}(\operatorname{cl}(A)) \cup$ $\operatorname{cl}(\operatorname{int}(A))$,

5. $t$-I-set [6] (resp. $t$-set [18]) if $\operatorname{int}\left(c l^{*}(A)\right)=\operatorname{int}(A)(\operatorname{resp} . \operatorname{int}(\operatorname{cl}(A))=\operatorname{int}(A))$,

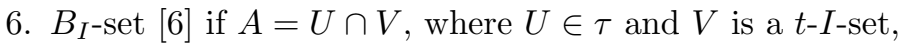

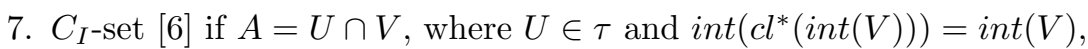

8. $A_{I}$-set [11] if $A=U \cap V$, where $U \in \tau$ and $V=(\operatorname{int}(V))^{*}$, 
9. strongly pre-I-open [17] if $A$ is pre- $I$-open as well as a $C_{I^{-}}$-set,

10. strongly $b$ - $I$-open [4] if $A$ is $b$ - $I$-open as well as a $C_{I}$-set and

11. $I$-locally closed set [5] if $A=U \cap V$, where $U \in \tau$ and $V=V^{*}$.

Definition 2.2. A subset $A$ of an ideal topological space $(X, \tau, I)$ is said to be $I$-nowhere dense if $\operatorname{int}\left(c l^{*}(A)\right)=\phi$.

Observe that if $A$ is rare, then a $t$ - $I$-set (resp. $t$-set) is $I$-nowhere dense (resp. nowhere dense). Recall that a set $A$ of $X$ is rare if it has no interior points. Also notice that if $A$ is rare, then $b$ - $I$-open sets and $b$-open sets are pre- $I$-open and preopen, respectively.

Definition 2.3. A function $f:(X, \tau, I) \rightarrow(Y, \sigma)$ is said to be

1. semi-I-continuous [6] if for every $V \in \sigma, f^{-1}(V)$ is semi-I-open,

2. pre-I-continuous [5] if for every $V \in \sigma, f^{-1}(V)$ is pre-I-open,

3. b-I-continuous [3] if for every $V \in \sigma, f^{-1}(V)$ is $b$ - $I$-open,

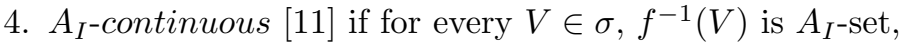

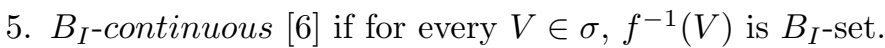

6. I-locally continuous [5] if for every $V \in \sigma, f^{-1}(V)$ is $I$-locally closed,

7. strongly pre-I-continuous [17] if for every $V \in \sigma, f^{-1}(V)$ is strongly pre-I-open and

8. strongly $b$-I-continuous [4] if for every $V \in \sigma, f^{-1}(V)$ is strongly $b$ - $I$-open.

\section{$3 \quad$ Stongly $\alpha-I$-Open Sets}

Definition 3.1. A subset $A$ of an ideal space $(X, \tau, I)$ is said to be strongly $\alpha$ - $I$-open set if $A$ is b-I-open as well as a $B_{I}$-set.

The family of all strongly $\alpha$-I-open sets in $(X, \tau, I)$ is denoted by $S$ - $\alpha I O(X, \tau)$ or $S$ - $\alpha I O(X)$. For a subset $A$ of $(X, \tau, I)$, int $t_{s \alpha}(A)=\bigcup\{U \subset A, U \in S$ - $\alpha I O(X, \tau)\}$. Clearly $\tau \subset S$ - $\alpha I O(X) \subset$ $\alpha I O(X)$. The following examples 3.2 and 3.3 show that these inclusions are not reversible.

Example 3.2. Let $X=\{a, b, c, d\}, \tau=\{X, \phi,\{a\},\{c\},\{a, c\}\}$ and $I=\{\phi,\{a\}\}$. If $A=\{c\}$, then $A^{*}=\{b, c, d\}$ and so $\operatorname{int}\left(c l^{*}(\operatorname{int}(A))\right)=\operatorname{int}\left(c l^{*}(\{c\})\right)=\operatorname{int}(\{b, c, d\})=\{c\}=A$. Therefore $A$ is $\alpha$-I-open. Since $X$ is the only open set containing $A, A=X \cap A$ is the only possibility to write $A$ as the intersection with $X$. Since $\operatorname{int}\left(c l^{*}(A)\right)=\operatorname{int}\left(c l^{*}(\{c\})\right)=\operatorname{int}(\{b, c, d\})=\operatorname{int}(A)$. This shows that $A$ is a $B_{I}$-set and hence $A$ is strongly $\alpha$-I-open set, but $A$ is not $I$-open. This shows 
the existence of non trivial strongly $\alpha$-I-open sets.

Example 3.3. Let $X=\{a, b, c, d\}, \tau=\{X, \phi,\{a\},\{c\},\{a, c\}\}$ and $I=\{\phi,\{a\}\}$. If $A=\{a, b, c\}$, then $A^{*}=\{a, c, d\}$ and so $\operatorname{int}\left(c l^{*}(\operatorname{int}(A))\right)=\operatorname{int}\left(c l^{*}(\{a, c\})\right)=\operatorname{int}(X)=X \supset A$. Therefore $A$ is $\alpha$-I-open. Since $X$ is the only open set containing $A, A=X \cap A$ is the only possibility to write $A$ as the intersection with $X$. Since $\operatorname{int}\left(c l^{*}(A)\right)=\operatorname{int}\left(c l^{*}(\{a, b, c\})\right)=\operatorname{int}(X)=X \neq \operatorname{int}(A), A$ is not a $B_{I}$-set and hence $A$ is not a strongly $\alpha$ - $I$-open set.

The following example shows that $\alpha$-I-open sets and $B_{I}$ sets are independent concepts.

Example 3.4. Consider the ideal space $(X, \tau, I)$ of Example 3.3.

(a) If $A=\{a, b, d\}$, then $\operatorname{int}(A)=\{a\}$ and $\operatorname{int}\left(c l^{*}(\operatorname{int}(A))\right)=\operatorname{int}\left(c l^{*}(\{a\})\right)=\operatorname{int}(\{a\}) \operatorname{does}$ not contains $A$. Therefore $A$ is not a $\alpha$-I-open set. But $\operatorname{int}\left(c l^{*}(A)\right)=\operatorname{int}\left(c l^{*}(\{a, b, d\})\right)=$ $\operatorname{int}(\{a, b, d\})=\{a\}=\operatorname{int}(A)$ and $A=X \cap A$. Therefore $A$ is a $B_{I^{-}}$set.

(b) If $B=\{a, b, c\}$, then $\operatorname{int}(B)=\{a, c\}$ and so $\operatorname{int}\left(c l^{*}(\operatorname{int}(B))\right)=\operatorname{int}\left(c l^{*}(\{a, c\})\right)=X \supset B$.

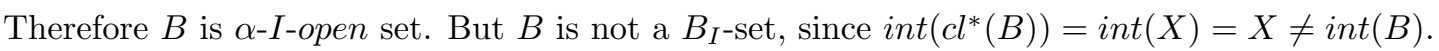

Theorem 3.5. Every strongly $\alpha$-I-open set is strongly pre-I-open.

Proof. It follows from the fact that every $\alpha$-I-open set is pre- $I$-open and let $A$ be a $B_{I}$ set. Then $A=U \cap V$, where $U \in \tau$ and $V$ is a $t$ - $I$-set. Then $\operatorname{int}(V)=\operatorname{int}\left(c l^{*}(V)\right) \supset \operatorname{int}\left(\operatorname{cl} l^{*}(\operatorname{int}(V))\right) \supset \operatorname{int}(V)$ and hence $\operatorname{int}(V)=\operatorname{int}\left(c l^{*}(\operatorname{int}(V))\right)$. This shows that $A$ is a $C_{I^{-}}$-set. Therefore $A$ is strongly preI-open set.

The converse of the above theorem need not be true by the following example.

Example 3.6. Consider $R$, the set of all real numbers with the usual topology and the ideal $I_{f}$ consisting of all finite subsets of $R$. If $A=Q$, the set of all rational numbers, then $A^{*}=$ $R$. Since $\operatorname{int}\left(c l^{*}(A)\right)=R \supset A, A$ is pre-I-open. Since $A=R \cap A$ where $R$ is open and $\operatorname{int}\left(c l^{*}(\operatorname{int}(A))\right)=\phi=\operatorname{int}(A)$, it follows that $A$ is strongly pre-I-open but $A$ is not strongly $\alpha$-I-open, since $\operatorname{int}\left(c l^{*}(\operatorname{int}(A))\right)=\phi$ does not contains $A$.

Theorem 3.7. Every strongly $\alpha$ - $I$-open set is strongly $b$ - $I$-open.

Proof. It follows from Theorem 3.5. and [4, Theorem 3.7].

The converse of the above theorem need not be true by the following example.

Example 3.8. Let $X=\{a, b, c\}, \tau=\{X, \phi,\{a\},\{b\},\{a, b\}\}$ and $I=\{\phi,\{b\}\}$. Then $A=\{a, c\}$ is strongly $b$ - $I$-open, but it is not strongly $\alpha$-I-open. For $\operatorname{int}\left(c l^{*}(A)\right) \cup c l^{*}(\operatorname{int}(A))=\operatorname{int}\left(\{a, c\}^{*} \cup\right.$ $\{a, c\}) \cup c l^{*}(\{a\})=\{a, c\} \supset A$. Therefore, $A$ is $b$-I-open. Since $X$ is the only open set containing $A, A=X \cap A$ is the only possibility to write $A$ as the intersection with $X$. Since $\operatorname{int}\left(c l^{*}(\operatorname{int}(A))\right)=\operatorname{int}\left(c l^{*}(\{a\})\right)=\operatorname{int}(\{a, c\})=\operatorname{int}(A)$ and hence $A$ is strongly $b$-I-open set. Since $\operatorname{int}\left(c l^{*}(\operatorname{int}(A))\right)=\operatorname{int}\left(c l^{*}(\{a\})\right)=\operatorname{int}(\{a, c\})=\{a\}$ is not contains $A$. Hence $A$ is not 
strongly $\alpha$-I-open.

Proposition 3.9. Let $(X, \tau, I)$ be an ideal topological space. A subset $A$ of $X$ is $I$-locally closed set if $A$ is both open and $A_{I \text {-set. }}$

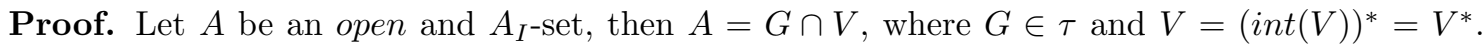
This shows that $A$ is $I$-locally closed set.

Observe that if $V$ is rare, then $A$ is empty.

The following theorem gives a characterization of open sets in terms of strongly $\alpha$ - $I$-open sets and $A_{I}$-sets.

Theorem 3.10. If $(X, \tau, I)$ is an ideal topological space. For a subset $A$ of $X$, the following conditions are equivalent.

(a) A is open.

(b) $A$ is open, strongly $\alpha-I$-open and $A_{I}$-set.

(c) $A$ is strongly $\alpha-I$-open and I-locally closed set.

(d) A is strongly pre-I-open and I-locally closed set.

(e) $A$ is strongly pre-I-open and $A_{I^{-}}$set.

\section{Proof.}

(a) $\Rightarrow(\mathrm{b})$ is obvious.

(b) $\Rightarrow$ (c) It follows from Proposition 3.9.

(c) $\Rightarrow$ (d) It follows from Theorem 3.5.

(d) $\Rightarrow$ (e) If $A$ is $I$-locally closed, then $A=G \cap A^{*}$ for some open set $G$. Since $A \subset A^{*}$, by [17, Lemma 2.5] $A^{*}=c l^{*}(A)$. Now $A \subset \operatorname{int}\left(c l^{*}(A)\right)=\operatorname{int}\left(A^{*}\right)$ and so $A^{*} \subset\left(\operatorname{int}\left(A^{*}\right)\right)^{*} \subset\left(A^{*}\right)^{*} \subset A^{*}$. Therefore $A^{*}=\left(\operatorname{int}\left(A^{*}\right)\right)^{*}$ which implies that $A$ is an $A_{I}$ set.

(e) $\Rightarrow$ (a) Suppose $A$ is strongly pre- $I$-open and $A_{I}$-set.

$$
\begin{aligned}
A & \subset \operatorname{int}\left(c l^{*}(A)\right) \\
& =\operatorname{int}\left(c l^{*}(U \cap V)\right)
\end{aligned}
$$

where $U$ is open and $V=(\operatorname{int}(V))^{*}$. By [10, Theorem 2.1.]

$$
\begin{aligned}
A & \subset U \cap\left(\operatorname{int}\left(c l^{*}(V)\right)\right) \\
& \subset U \cap \operatorname{int}\left(V^{*}\right) \\
& \subset U \cap \operatorname{int}(\operatorname{int}(V))^{*} \\
& \subset U \cap \operatorname{int}\left(c l^{*}(\operatorname{int}(V))\right) \\
& \subset U \cap \operatorname{int}(V) \\
& =\operatorname{int}(A)
\end{aligned}
$$


Theorem 3.11. Let $(X, \tau, I)$ be an ideal topological space. A subset $A$ of $(X, \tau, I)$ is pre-I-open and $B_{I}$-set if $A$ is strongly $\alpha-I$-open.

Proof. Let $A$ be strongly $\alpha$ - $I$-open set. Since every $\alpha$ - $I$-open set is pre-I-open, then $A$ is pre-Iopen and $B_{I^{-} \text {-set. }}$

Theorem 3.12. Let $(X, \tau, I)$ be an ideal topological space. A subset $A$ of $(X, \tau, I)$ is strongly $\alpha-I$-open if and only if it is semi-I-open, pre-I-open and $B_{I}$-set.

Proof.

Necessity. It follows from the fact that every $\alpha$-I-open set is semi-I-open and pre-I-open.

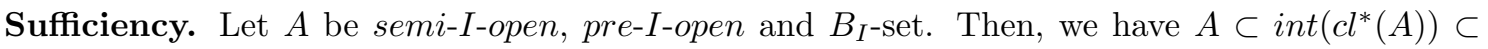
$\operatorname{int}\left(c l^{*}\left(c l^{*}(\operatorname{int}(A))\right)\right)=\operatorname{int}\left(c l^{*}(\operatorname{int}(A))\right)$. This shows that $A$ is $\alpha$-I-open set and also $A$ is $B_{I^{-}}$-set. Therefore $A$ is a strongly $\alpha$ - $I$-open set.

\section{Strongly $\alpha$ - $I$-Ccontinuous Maps}

Definition 4.1. A mapping $f:(X, \tau, I) \rightarrow(Y, \sigma)$ is said to be strongly $\alpha$ - $I$-continuous if for every $V \in \sigma, f^{-1}(V)$ is strongly $\alpha$-I-open.

Theorem 4.2. Every strongly $\alpha$-I-continuous map is strongly pre-I-continuous.

Proof. It follows from Theorem 3.5.

Theorem 4.3. Every strongly $\alpha$-I-continuous map is strongly b-I-continuous.

Proof. It follows from Theorem 3.7.

Theorem 4.4. Let $f:(X, \tau, I) \rightarrow(Y, \sigma)$ be any mapping. Then $f$ is $I$-locally continuous map if it is both continuous and $A_{I}$-continuous.

Proof. It follows from Proposition 3.9.

Theorem 4.5. Let $f:(X, \tau, I) \rightarrow(Y, \sigma)$ be any mapping. Then the following conditions are equivalent.

(a) $f$ is continuous.

(b) $f$ is continuous, strongly $\alpha-I$-continuous and $A_{I}$-continuous.

(c) $f$ is strongly $\alpha$-I-continuous and I-locally continuous.

(d) $f$ is strongly pre-I-continuous and I-locally continuous.

(e) $f$ is strongly pre-I-continuous and $A_{I}$-continuous.

Proof. It follows from Theorem 3.10. 
Theorem 4.6. Let $f:(X, \tau, I) \rightarrow(Y, \sigma)$ be any mapping. Then $f$ is pre-I-continuous and $B_{I}$-continuous if $f$ is strongly $\alpha$-I-continuous.

Proof. It follows from Theorem 3.11.

Theorem 4.7. Let $f:(X, \tau, I) \rightarrow(Y, \sigma)$ be any mapping. Then $f$ is strongly $\alpha$-I-continuous if and only if it is semi-I-continuous, pre-I-continuous and $B_{I}$-continuous.

Proof. It follows from Theorem 3.12. Definition 4.8. A mapping $f:(X, \tau, I) \rightarrow(Y, \sigma, I)$ is said to be strongly $\alpha$ - $I$-irresolute if $f^{-1}(V)$ is strongly $\alpha$-I-open in $X$ for every strongly $\alpha$ - $I$-open set $V$ of $Y$.

Theorem 4.9. Let $f:(X, \tau, I) \rightarrow(Y, \sigma)$ and $g:(Y, \sigma) \rightarrow(Z, \eta)$ be mappings. Then the composition $g \circ f: X \rightarrow Z$ is strongly $\alpha-I$-continuous if $g$ is continuous and $f$ is strongly $\alpha-I$-continuous. Proof. Let $W$ be any open subset of $Z$. Since $g$ is continuous, $g^{-1}(W)$ is open in $Y$. Since $f$ is strongly $\alpha$ - $I$-continuous, then $(g \circ f)^{-1}(W)=f^{-1}\left(g^{-1}(W)\right)$ is strongly $\alpha$ - $I$-open in $X$ and hence $g \circ f$ is strongly $\alpha$ - $I$-continuous.

Theorem 4.10. Let $f:\left(X, \tau, I_{1}\right) \rightarrow\left(Y, \sigma, I_{2}\right)$ and $g:\left(Y, \sigma, I_{2}\right) \rightarrow\left(Z, \eta, I_{3}\right)$ be mappings. Then the composition $g \circ f: X \rightarrow Z$ is strongly $\alpha$-I-continuous if $g$ is strongly $\alpha$-I-continuous and $f$ is strongly $\alpha$-I-irresolute.

Proof. Let $W$ be any open subset of $Z$. Since $g$ is strongly $\alpha$-I-continuous, $g^{-1}(W)$ is strongly $\alpha$ - $I$-open in $Y$. Since $f$ is strongly $\alpha$-I-irresolute, then $(g \circ f)^{-1}(W)=f^{-1}\left(g^{-1}(W)\right)$ is strongly $\alpha$-I-open in $X$ and hence $g \circ f$ is strongly $\alpha$-I-continuous.

Theorem 4.11. Let $f:\left(X, \tau, I_{1}\right) \rightarrow\left(Y, \sigma, I_{2}\right)$ and $g:\left(Y, \sigma, I_{2}\right) \rightarrow\left(Z, \eta, I_{3}\right)$ be mappings. Then the composition $g \circ f: X \rightarrow Z$ is strongly $\alpha$-I-irresolute if both $f$ and $g$ are strongly $\alpha$-I-irresolute. Proof. Let $W$ be any strongly $\alpha$ - $I$-open subset of $Z$. Since $g$ is strongly $\alpha$ - $I$-irresolute, $g^{-1}(W)$ is strongly $\alpha$-I-open in $Y$. Since $f$ is strongly $\alpha$-I-irresolute, then $(g \circ f)^{-1}(W)=f^{-1}\left(g^{-1}(W)\right)$ is strongly $\alpha$-I-open in $X$ and hence $g \circ f$ is strongly $\alpha$-I-irresolute.

Definition 4.12. [15] Let $A$ be a subset of a space $(X, \tau)$ then the set $\cap\{U \in \tau: A \subset U\}$ is called the kernel of $A$ and denoted by $\operatorname{ker}(A)$.

Lemma 4.13. [7] Let $A$ be a subset of a space $(X, \tau)$, then

(a) $x \in \operatorname{ker}(A)$ if and only if $A \cap F \neq \phi$ for any closed subset $F$ of $X$ with $x \in F$;

(b) $A \subset \operatorname{ker}(A)$ and $A=\operatorname{ker}(A)$ if $A$ is open in $X$;

(c) if $A \subset B$, then $\operatorname{ker}(A) \subset \operatorname{ker}(B)$.

Definition 4.14. Let $N$ be a subset of a space $(X, \tau, I)$ and $x \in X$. Then $N$ is called strongly $\alpha$ - $I$-neighbourhood of $x$, if there exists a strongly $\alpha$ - $I$-open set $U$ containing $x$ such that $U \subset N$. 
Theorem 4.15. The following statements are equivalent for a mapping $f:(X, \tau, I) \rightarrow(Y, \sigma)$.

1. $f$ is strongly $\alpha$-I-continuous.

2. for each $x \in X$ and each open set $V$ in $Y$ with $f(x) \in V$, there exists a strongly $\alpha$-I-open set $U$ containing $x$ such that $f(U) \subset V$.

3. for each $x \in X$ and each open set $V$ in $Y$ with $f(x) \in V, f^{-1}(V)$ is a strongly $\alpha-I$ neighbourhood of $x$.

\section{Proof.}

(1) $\Rightarrow(2)$ Let $x \in X$ and $V$ be an open set in $Y$ such that $f(x) \in V$. Since $f$ is strongly $\alpha$-I-continuous, $f^{-1}(V)$ is a strongly $\alpha$-I-open containing $x$. Set $U=f^{-1}(V)$. Then we have $f(U) \subset V$.

$(2) \Rightarrow(3)$ Let $V$ be an open set in $Y$ and let $f(x) \in V$. Then by (2), there exists a strongly $\alpha$-I-open set $U$ containing $x$ such that $f(U) \subset V$. So $x \in U \subset f^{-1}(V)$. Hence $f^{-1}(V)$ is a strongly $\alpha-I$-neighbourhood of $x$.

$(3) \Rightarrow(1)$ Let $V$ be an open set in $Y$ and let $f(x) \in V$ then by $(3), f^{-1}(V)$ is a strongly $\alpha-I$ neighbourhood of $x$. Thus for each $x \in f^{-1}(V)$ there exists a strongly $\alpha$-I-open set $U_{x}$ containing $x$ such that $x \in U_{x} \subset f^{-1}(V)$. Hence $f^{-1}(V) \subset \cup_{x \in f^{-1}(V)} U_{x}$ so $f^{-1}(V) \in S-\alpha I O(X)$.

Theorem 4.16. The following mappings are equivalent for a mapping $f:(X, \tau, I) \rightarrow(Y, \sigma)$.

1. $f$ is strongly $\alpha$-I-continuous.

2. for every subset $A$ of $X, f\left(\right.$ int $\left._{s \alpha} I(A)\right) \subset \operatorname{ker}(f(A))$.

3. for every subset $B$ of $Y$, int $t_{s \alpha} I\left(f^{-1}(B)\right) \subset f^{-1}(\operatorname{ker}(B))$.

\section{Proof.}

$(1) \Rightarrow(2)$ Let $A$ be any subset of $X$. Suppose that $y \notin k e r(f(A))$. Then by Lemma 4.13. there exists a closed subset $F$ of $Y$ such that $y \in F$ and $f(A) \cap F=\phi$. Thus we have $A \cap f^{-1}(F)=\phi$ and $\left(\right.$ int $\left._{s \alpha}(I(A))\right) \cap f^{-1}(F)=\phi$. Therefore, we obtain $f\left(\right.$ int $\left._{s \alpha}(I(A))\right) \cap F=\phi$ and $y \notin f\left(\right.$ int $\left._{s \alpha} I(A)\right)$. This implies that $f\left(\right.$ int $\left._{s \alpha} I(A)\right) \subset \operatorname{ker}(A)$.

$(2) \Rightarrow(3)$ Let $B$ be any subset of $Y$ by (2) and Lemma 4.13., we have $f\left(\operatorname{int}_{s \alpha} I\left(f^{-1}(B)\right)\right) \subset$ $\operatorname{ker}\left(f\left(f^{-1}(B)\right)\right) \subset \operatorname{ker}(B)$ and $\operatorname{int}_{s \alpha} I\left(f^{-1}(B)\right) \subset f^{-1}(\operatorname{ker}(B))$.

$(3) \Rightarrow(1)$ Let $V$ be an open set of $Y$. Then by Lemma 4.13. and (3), we have $\operatorname{int}_{s \alpha} I\left(f^{-1}(V)\right) \subset$ $f^{-1}(k e r(V))=f^{-1}(V)$ and $\operatorname{int}_{s \alpha} I\left(f^{-1}(V)\right)=f^{-1}(V)$. This implies $f^{-1}(V)$ is strongly $\alpha$-I-open.

Received: April 2009. Revised: August 2009. 


\section{References}

[1] M.E. Abd El-Monsef, E.F.Lashien And A.A. Nasef, On I-open sets and I-continuous mappings, Kyungpook Mathematical Journal, Vol. 32, No. 1 (1992), 21-30.

[2] D. Andrijevic, On b-open sets, Mathematichki Vesnik, Vol.48, No. 1-2 (1996), 59-64.

[3] A. Caksu Guler and G. Aslim, b-I-open sets and decomposition of continuity via idealization, Proceedings of Institute of Mathematics and Mechanics. National Academy of Sciences of Azerbaijan, Vol. 22 (2005), 27-32.

[4] R. Devi, A. Selvakumar and M. Parimala, Strongly b-I-open sets in ideal topological spaces, (submitted).

[5] J. Dontchev, Idealization of Ganster-Reilly decomposition theorems, (1999), http://arxiv.org/abs/Math.GN/9901017.

[6] E. Hatir And T. Noiri, On decomposition of continuity via idealization, Acta Math. Hungar., 96 (4) (2002), 341-349.

[7] S. Jafari and T. Noiri, Contra-super-continuous mappings, Annales Universitatis Scientiarum Budapestinensis, Vol. 22 (1999), 27-34.

[8] D. Jankovic and T.R. Hamlett, Compatible extensions of ideals, Unione Matematica Italiana Bollettino. B. Serie VII, Vol. 6, No. 3 (1992), 453-465.

[9] D. Jankovic and T.R. Hamlett, New topologies from old via ideals, Amer. Math. Monthly, 97 (1990), 295-310.

[10] V. Jeyanthi, V. Renuka Devi and D. Sivaraj, Some subsets of ideal topological spaces, Math. Benchink, 59 (2007), 75-84.

[11] A. Keskin, T. Noiri And S. Yuksel, Idealization of decomposition theorem, Acta Math. Hungar., 102 (2004), 269-277.

[12] N. Levine, Semi-open sets and semi-continuity in topological spaces, Amer. Math. Monthly, 70 (1963), 36-41.

[13] A. S. Mashhour, M. E. Abd El-Monsef and S. N. El-Deeb, On precontinuous and weak precontinuous mappings, Proc. Math. Phys. Soc. Egypt, 53 (1982), 47-53.

[14] A. S. Mashrour, I. N. Hasanein and S. N. El-Deeb, $\alpha$-continuous and $\alpha$-open mappings, Acta Math. Hungar., 41 (1983), 213-218.

[15] M. Mrsevic, On Pairwise $R_{0}$ and pairwise $R_{1}$ bitopological spaces, Bulletin Mathematique de la Societe des Sciences Mathematiques de la Republique Socialiste de Roumanie. Nouvelle Serie, Vol. 30 (78), No. 2 (1986), 141-148. 
[16] O. NJastad, On some classes of nearly open sets, Pacific J. Math., 15 (1965), 961-970.

[17] V. Renuka Devi and D. Sivaraj, A Decomposition of continuity via ideals, Acta. Math. Hungar., Vol. 118 (1-2) (2008), 53-59.

[18] J. Tong, On decomposition of continuity in topological spaces, Acta Math. Hungar., 54 (1989), 51-55.

[19] R. Vaidyanathaswamy, The localisation theory in set topology, Proc. Indian Acad. Sci. Math. Sci., 20 (1945), 51-61. 